\title{
Summer School on Intelligent Agents in Automation: Hands-on Educational Experience on Deploying Industrial Agents
}

\author{
Paulo Leitão, ${ }^{1,2}$, Luís Ribeiro ${ }^{3}$, José Barata ${ }^{4}$, Birgit Vogel-Heuser ${ }^{5}$ \\ ${ }^{1}$ Polytechnic Institute of Bragança, Campus Sta Apolónia, 5300-253 Bragança, Portugal, pleitao@ipb.pt \\ ${ }^{2}$ LIACC - Artificial Intelligence and Computer Science Laboratory, Rua Campo Alegre 1021, 4169-007 Porto, Portugal \\ ${ }^{3}$ Linkoping University, SE-58 183 Linköping, Sweden, luis.ribeiro@liu.se \\ ${ }^{4}$ New University of Lisbon, Faculty of Sciences and Technology, Quinta da Torre, 2825-114 Caparica, Portugal, jab@uninova.pt \\ ${ }^{5}$ Technische Universität München, Boltzmannstraße 15, 85748 Garching near Munich, Germany vogel-heuser@ais.mw.tum.de
}

\begin{abstract}
Cyber-physical systems constitutes a framework to develop intelligent, distributed, resilient, collaborative and cooperative systems, promoting the fusion of computational entities and physical devices. Agent technology plays a crucial role to develop this kind of systems by offering a decentralized, distributed, modular, robust and reconfigurable control structure. This paper describes the implementation of a summer school aiming to enhance the participants' knowledge in the field of multi-agent systems applied to industrial environments, being able to gain the necessary theoretical and practical skills to develop real industrial agent based applications. This is accomplished in an international framework where individual knowledge and experiences are shared in a complementary level.
\end{abstract}

\section{INTRODUCTION}

The term cyber-physical system (CPS) has emerged relatively recently to denote the next generation of embedded systems. However, unlike this preceding generation, CPSs denote a much higher degree of integration between the cyber and the physical parts of the system. This is in principle an identity relationship that cannot or should not be dissociated. The cyber-representation acts effectively as the "mind" of the physical component. Furthermore, such relationship needs to be extended to all value-adding components in a system. These will interact through these autonomous and dynamic programming constructs instead of the traditional predetermined and rigid communication protocols. The possibility of designing and building systems based on such premises has motivated a global investment in research and development worldwide, not restricted but including Industrie 4.0 (Germany), Industrial Internet Consortium (US), Made in China 2025 (China) and Factories of the Future (EU) [1]. Such investment spans across several disciplines from traditional automation sectors such as factory and enterprise environments, building and home automation, and energy, to traditionally less automated sectors such agriculture and health [1][2].

The existing automation practices often result in bespoke solutions that are not applicable beyond the boundaries of a specific system. CPS design is necessarily more complex and challenging since the cyber-physical component should be able to operate on many different systems. Such systems will recognize the component and instantly interact with it. The component itself will provide a response based on its new operational context but also on its operational history. This means that a component should be considered as part of wider domain specific reference architectures that characterize the dynamic rules of engagement and interaction.

The act of connecting a cyber-physical component in a CPS supposes subsequently a plug and play connectivity that is established at the physical level but also the logical and cognitive levels which results in a tremendous integration effort that must be formalized in advanced [3].

Such aspects have been widely explored by the multi-agent systems (MAS) community and with particular emphasis in their application to factory automation. Agents are particularly adequate for the creation of the cyber-envelope of a cyberphysical component since by design they include qualities as autonomy, social-ability, intelligence, reactivity and pro-activity [4]. Several industrial prototypes in factory automation have demonstrated the potential of using agents and the virtues of CPS-based automation before the term CPS had been coined [5]. However, there have also been persisting barriers to the widespread adoption of agent technology [6] ranging from pure technological issues, such as the low degree of maturity of agent technology, to more socio-technical problems, including the lack of specialized technicians and practitioners that would master both the technical and scientific aspects of such emerging technologies. However, the required technologies have been maturing fast and there is an increased set of drivers for its adoption including the need to develop and adopt more sustainable production practices based on the intensive tracking and re-use of both products and systems.

There is nevertheless an important gap to fill concerning the education of future engineers. Despite the wide offer on summer schools (e.g., European Agent Systems Summer School) and doctoral workshops (e.g., AAMAS - Autonomous Agents and Multi-Agent Systems doctoral mentoring program) on the design of MAS, these have been mostly focused on traditional artificial intelligence (AI) aspects. Traditional AI is a 
fundamental discipline in the design of industrial automation systems, however there are other specific challenges that are not traditionally covered. In particular, the interconnectivity aspects and design principles that enable agents to operate in an industrial environment have been consistently lacking. The industrial automation world has been dominated by the use of programmable logic controllers (PLCs) and programming languages based on the IEC 61131-3 standards that collectively ensure the time critical response of the system. These extremely matured and proven technologies are expected to persist in the market at the least for the incoming decade. Hence it is fundamental to teach the future automation engineers how to use, modify and integrate such technologies with emerging CPS system design principles and technologies to indeed prepare the grounds for the development of the future production floors as envisioned by research programmes worldwide.

Having this in mind, the 1st International Summer School on Intelligent Agents in Automation, technically supported by the IEEE IES Technical Committee on Industrial Agents, was held in the campus of New University of Lisbon, Portugal, from $27^{\text {th }}$ to $30^{\text {th }}$ July 2015 . The objectives of the summer school were to enhance the participants' knowledge in the field of MAS applied to industrial environments (e.g., production systems and smart grids), being able to gain a range of theoretical and practical skills necessary to develop real industrial agent based applications. In contrast to similar summer schools, $\mathrm{PhD}$ tracks or colloquiums, which are held usually in conjunction with conferences, the main objective of the summer school was the practical experience on implementing agents. Particularly, the course introduces the MAS principles as a suitable paradigm to develop industrial distributed collaborative systems, and provide practical competences in developing and deploying MAS solutions for industrial automation applications. The topics were lectured by international, well-known researchers in the respective fields, being theory, applications and development procedures described. The state of the art and cutting edge research was presented through two invited industrial keynote speeches. A special emphasis was devoted to the hands-on exercise along the several modules, but particularly in a projectbased learning module devoted to the practical development of agent-based applications for automation systems (using real hardware automation devices).

The multi-disciplinary nature of the summer school, aligned with the Bologna process, also allows the exchange of expertise and learning practices in the field of industrial agents, creating an opportunity of each participant to introduce improvements in the current contents of their R\&D expertise.

The rest of the paper is organized as follows: Section II describes the organization of the summer school and Section III presents the contents of the lecturing modules. Section IV details the practical development of agent-based solutions for automation domain and Section $\mathrm{V}$ presents the results of the implementation of the described summer school and summarizes the achieved innovation and impact. Finally, Section VI rounds up the paper with the conclusions.

\section{Pedagogical Methodology}

The course on intelligent agents in automation took 4 days, comprising 7 workload hours per day, and was mainly addressed for advanced practitioners from industry and academia, and students of graduate-level courses (MSc and PhD courses). The lectures and didactical material used the English language.

The structure of the summer school comprised a set of lectures and tutorials, as illustrated in Fig. 1, held by well-known researchers in the field (and members of the IEEE IES Technical Committee on Industrial Agents). The welcome session constituted the opportunity to welcome the attendees and also to present the objective of the summer school, pedagogical methodologies and calendar of activities.

\begin{tabular}{|c|c|c|c|}
\hline $\begin{array}{c}\text { 27/07/2015 } \\
\text { Monday }\end{array}$ & $\begin{array}{c}28 / 07 / 2015 \\
\text { Tuesday }\end{array}$ & $\begin{array}{l}\text { 29/07/2015 } \\
\text { Wednesday }\end{array}$ & $\begin{array}{c}\text { 30/07/2015 } \\
\text { Thursday }\end{array}$ \\
\hline Welcome & \multirow{3}{*}{$\begin{array}{c}\text { Model-driven engineering } \\
\text { and implementation of } \\
\text { field level agents with IEC } \\
61131-3\end{array}$} & $\begin{array}{l}\text { Industrial Keynote: } \\
\text { Luis Flores (IntroSys) }\end{array}$ & \multirow{3}{*}{$\begin{array}{l}\text { Service-oriented cloud } \\
\text { based cyber-physical } \\
\text { systems in automation }\end{array}$} \\
\hline \multirow{2}{*}{$\begin{array}{c}\text { Fundamentals in agent- } \\
\text { orientation and holonic } \\
\text { paradigm }\end{array}$} & & $\begin{array}{l}\text { Industrial Keynote: } \\
\text { José Barata (Uninova) }\end{array}$ & \\
\hline & & $\begin{array}{l}\text { Practical development of } \\
\text { agent-based applications } \\
\text { for automation }\end{array}$ & \\
\hline Lunch & Lunch & Lunch & Lunch \\
\hline $\begin{array}{l}\text { Architectural Design, } \\
\text { Deployment and } \\
\text { Assesment }\end{array}$ & $\begin{array}{c}\text { Designing and } \\
\text { implementing field level } \\
\text { agents with IEC } 61499\end{array}$ & $\begin{array}{l}\text { Practical development of } \\
\text { agent-based applications } \\
\text { for automation }\end{array}$ & $\begin{array}{l}\text { Agent for smart grid } \\
\text { applications }\end{array}$ \\
\hline
\end{tabular}

Fig. 1. Schedule of lectures for the summer school.

The technical modules covered different topics of applying intelligent agents to industrial domains, namely providing fundamental knowledge about design, development and deployment of agent-based solutions, constituting an introduction to this field. Advanced topics were related to deploying agents in the field level, and particularly interconnecting with IEC 61131-3 and IEC 61499, and also for developing cyber-physical systems that combine agent technology with service-oriented architectures (SOA) and cloud computing. An example of application of agent technology for smart grids applications is also lectured.

Each module is coordinated by one expert, providing knowledge and expertise in a particular topic, allowing to bring complementary knowledge and expertise in the field. In some modules, different participants have collaborated in the lecturing activities aiming to combine synergies and knowledge.

The teaching and learning methodology was based in classroom sessions, where the topics were presented using multimedia facilities, such as power-point slides. Particularly, demonstrations, simulations and videos were used to show, in a realistic way, the addressed topics. Each module had a duration of 3,5 hours and comprised the following components:

- A theoretical part, with a duration of 1 hour, aiming to provide theory and basics in the addressed topic.

- A practical part, with a duration of 2,5 hours, aiming the realization of exercises for the practical development of agent-based applications for automation, using e.g., the JADE framework. In this case, the practice was introduced during 30 minutes, and subsequently, was exercised during 2 hours.

The practical works aimed to provide hands-on competences and consolidate the acquired learning, being the developed works performed in groups of 2 - 3 students. A project-based 
learning practice was included in the summer course to complement the knowledge acquired during the theoretical and practical lectures, re-enforcing the acquisition of hands-on skills in developing agent-based solutions for automation domain. Aligned with the Bologna principles, the non-presential component assumed a crucial importance for the success of the learning process, and consequently, students were motivated to complement the development of laboratorial works (e.g., by addressing more advanced challenges) in non-presential hours.

The set of lectures was complemented with 2 industrial keynotes about practical aspects of presented topics to get in touch with industry requirements, practices and experiments.

An e-learning platform was used to support the pedagogical activities during the course, contributing for a sustainable dissemination and sharing of knowledge. The e-learning platform hosted the learning materials, namely lecture notes, exercises and laboratorial demonstrations.

\section{COURSE CONTENTS}

This section details the description of the contents of each module included in the summer school.

\section{A. Fundamentals in Agent-orientation and Holonic Paradigm}

The objective of this module was to provide an introduction to the main concepts of agent-based systems and holonic systems, as well as the available technologies and frameworks to develop such systems. A special attention was devoted to the development of practical agent-based applications using the JADE framework following a project-based learning. In detail, topics of this course comprised:

- Introduction to MAS: definition of agent and MAS, agent architectures, holonic principles, modelling individual behaviors, interaction protocols and ontologies.

- Frameworks to develop agent-based solutions: functionalities of agent-based frameworks, existing frameworks, introduction to the JADE framework [7].

- Implementation of practical examples using JADE: step by step learning with guidance of students, and implementation of advanced functionalities.

This module was lectured by Paulo Leitão and José Barbosa, both from Polytechnic Institute of Bragança, Portugal.

\section{B. Architectural Design, Deployment and Assessment}

The objective of this module was to provide an introduction to the design, deployment and assessment of agent-based architectures for cyber-physical production systems. The module focused on characterizing the different strategies to design agent based architectures and on the importance of proper design of agent architectures in a self-organization context. In detail, topics of this course comprised:

- Introduction to the design of multi-agent based architectures for CPS: design trends, advantages, limitations and tradeoffs, and strategies for the deployment of cyber-physical production systems.

- Assessment of MAS at architecture and system levels.
- Experimenting practically with different interaction patterns in JADE and assessing the scalability limits.

- Learning how to build a simple Hardware Abstraction Layer (HAL) in JADE/JAVA.

- Deployment of robust agent-based systems with JADE.

The module was lectured by Luis Ribeiro (Linköping University, Sweden) and João Ferreira (Royal Institute of Technology, Sweden).

\section{Model-driven Engineering and Implementation of Field Level Agents with IEC 61131-3}

The industrial production domain is recently subject to two major trends: firstly, increasing dynamics of global markets forces industrial enterprises to ensure the adaptability and robustness of their automated production systems. Today's concepts for implementing field level automation software is mostly realized on programmable logical controllers according to the IEC 61131-3 standard [8] which provides limited, inherent flexibility concepts. In contrast, agent-based concepts for designing and implementing field level software provide more flexibility. Combining agent concepts and IEC 61131-3 led to intelligent, flexible field level automation software. Secondly, rising complexity of (intelligent, flexible) automated production systems require concepts for supporting automation engineers in handling this complexity. In this context, the model-driven engineering paradigm emerged from the effort for separation of specification and implementation tasks [9].

These trends were addressed within this course focusing on the model-driven engineering of intelligent field level agents for automated production systems which can operate on IEC 611313 control hardware. In detail, topics of this course comprised:

- Fundamentals in model-driven engineering of automated production systems and implementing field level software in IEC 61131.

- Implementation of field level MAS for IEC 61131 environments.

- Model-based development of field level agents and their knowledge bases.

- Automatic generation of field level agents' executable field level code.

The module was lectured by Birgit Vogel-Heuser (Technische Universität München, Germany).

\section{Designing and Implementing Field Level Agents with IEC 61499}

This module discussed the usage of IEC 61499 for the field level agent design. The amount of control software which is required in modern industrial automation is drastically increasing. While on the higher levels sophisticated design and development methods, tools, and languages are already established, on the lower levels they are still partly missing. In order to improve this situation, the IEC 61499 reference model for distribution automation [10] has been introduced. On one hand, it targets a more formal way of modeling control software using a component-oriented design approach, and on the other 
hand it addresses important requirements (distribution and reconfiguration) for the lower field levels which are not targeted by classic control software development approaches. In detail, topics of this course comprised:

- An introduction to the IEC 61499 reference models.

- An overview of open source solutions for IEC 61499, including the 4DIAC framework [11].

- Usage and hands on examples for implementing own control applications in IEC 61499.

- Implementing the control application of a laboratory production station.

The module was lectured by Thomas Strasser (AIT Austrian Institute of Technology, Austria).

\section{E. Service-oriented Cloud-based Cyber-Physical Systems in Automation}

This module intent to provide hands-on knowledge on SOA principles and on its application to specify, develop and implement cloud-based automation and control/management systems. For this purpose, the module included an introduction to the major characteristics of SOA-methodologies, -techniques and -tools, and presented lessons learned from the implemented industrial prototypes, particularly in manufacturing and process automation domains. In detail, topics of this course comprised:

- Introduction to SOA and cloud-based automation: definition and characteristics.

- Migrating from ISA'88- and ISA'95-compliant automation architectures to SOA and cloud-based infrastructures. Illustration with industrial examples.

- Description of a practical example: step by step learning with guidance of students.

The module was lectured by Armando Walter Colombo (Hochschule Emden/Leer).

\section{F. Agent for Smart Grid Applications}

A smart grid is a complex energy exchange network with distributed energy generation, which motivates bi-directional flow of energy. The complexity of the smart grid control can be managed by a network of distributed agents performing intelligent control to achieve local goals and participating in the overall smart grid operation and control to achieve the system objectives. The proposed agent's architecture is aimed at facilitating the industrial adoption of the agent technology, reflecting the concepts of power system automation domain and based on IEC 61499 and IEC 61850 industrial standards. This architecture builds on the Logical Node concept of IEC 61850 and comprises deliberative and reactive layers, combining the advantages of both. By relying on the underlined IEC 61850 and IEC 61499 industrial standards, the architecture ensures the practical applicability and captures the domain specific concepts in the agent-based system design.

This module introduced the proposed agent's architecture and its theoretical foundation, being discussed the hybrid architecture structure based on reactive and deliberative layers. The practical part of the tutorial offers exercises on creating an agent in IEC 61499 IDE from the IEC 61850 system specification, namely the identification and modelling of agents, the developing of the agent behavior, the creation of the intention stack, interpreter and plans for the deliberative layer, and the behavior modules for the reactive layer. Finally, the developed agent based system is deployed to softPLCs.

The module was lectured by Gulnara Zhabelova (Luleå University of Technology, Sweden).

\section{G. Industrial Keynotes}

In addition to the technical modules, two keynotes were provided by experts in using agent technology in industry. The first one, provided by Luis Flores, CEO of Introsys, discussed the experience of using agents in industrial environments and particularly in modular and reconfigurable robotic systems for automotive industry. The second keynote was lectured by Jose Barata from UNINOVA, which presented the experience of his R\&D group in applying the agent technology in different industrial environments within several FP7 and H2020 European projects, namely IDEAS [12] and PRIME [13].

\section{Practical DeVelopment of Agent-Based APPLICATIONS FOR AUTOMATION DOMAIN}

The described modules were complemented by a module related to the practical development of agent-based applications in automation, where the attendees can apply and consolidate the acquired knowledge following a project based learning approach. An automation challenge was proposed to the students considering the development of a modular transfer system, as illustrated in Fig. 2.

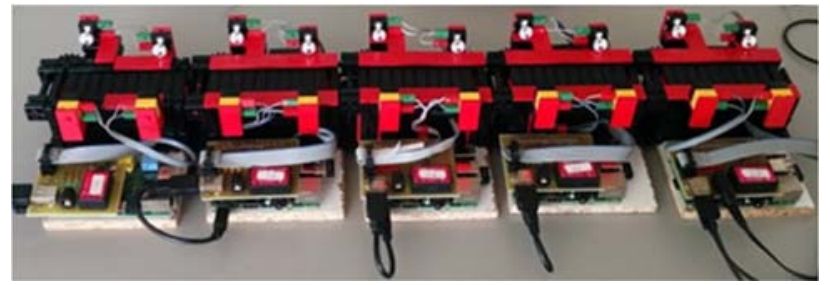

Fig. 2. Automation hardware setup.

The transfer system is composed by the sequential arrangement of several individual transfer systems based on individual Fischertechnik conveyors, being possible to transfer a part from an initial input location to an output location. For this purpose, the individual transfer systems are physically interconnected and at the logic control level, the agents need to interact to achieve the control of the global system.

Each group is responsible to develop an agent to be embedded in a Raspberry PI to perform the logic control of the individual transfer system: i.e. when requested and the part is detected, the conveyor system should perform the transfer of the part to the output destination. For this purpose, it is required that the agent accesses to the input IR sensor that detects the presence of a part in the input location, to the output IR sensor that detects the part in the output location, and to the motor that allows to transfer the part. At the end, the package comprising the agent running in the Raspberry $\mathrm{Pi}$ and the Fischertechnik conveyor constitutes a cyber-physical component. 
The global behavior of the modular transfer system emerges from the interaction among the distributed agents, developed by each group for each individual transfer system. For this purpose, the interaction patterns for exchanging messages among agents were designed and implemented by the several groups, as well as a basic agreement on the semantics used for the exchange of the message's contents.

In an initial phase, the configuration of the transfer system was pre-defined, using a XML file, with each agent knowing what are the precedent and posterior transfer systems, and interacting with the agents representing these devices. In an advanced stage, the re-configuration was performed automatically and the agents were able to discover the precedent and posterior transfer systems by interacting with the agents.

The accomplishment of this module involved a strong practical component related to designing, programing and deploying agents in automation environments comprising the physical hardware devices, allowing the attendees to acquire solid hands-on skills in this field.

\section{IMPLEMENTATION OF THE SUMMER SCHOOL}

The summer school was held in the campus of New University of Lisbon, Portugal, from $27^{\text {th }}$ to $30^{\text {th }}$ July 2015 . The attendance comprised 18 students coming from 9 different countries, and 9 teachers coming from 4 different countries, as summarized in Fig. 3.

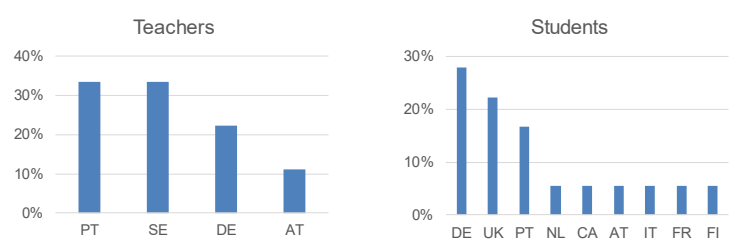

Fig. 3. Characterization of the attendance to the summer school.

The analysis of the course's implementation, and particularly the innovation and impact, will be detailed in the next sections.

\section{A. Analysis of the Feedback from Students}

The execution of the summer school was evaluated taking into consideration the feedback from the different players in the project. For this purpose, the students were invited to answer an individual questionnaire about, amongst others, the course structure and organization, pedagogical and didactic activities. The questionnaire uses the Likert scale for the majority of answers, i.e., (1) Not at all satisfied, (2) Not very satisfied, (3) Fairly satisfied, (4) Very satisfied, and (5) Very much satisfied.

The feedback from the students showed that they were highly motivated, and at the end, they gained confidence in deploying agent technology. Namely, $68,8 \%$ of the students were very or very much satisfied with the duration of the course and 93,8 \% with the dates, which reinforces the benefits of having this kind of learning events concentrated in a short period. In fact, the majority of students pointed out 4 or 5 days as the best duration for the summer school.

The factors that mostly motivated the participation of the students in the course were mainly to learn advanced features of agents in automation ( $93,8 \%$ answered very or very much), practice the deployment of industrial agents $(81,3 \%)$ and be introduced to agent-based systems $(50 \%)$. This motivation is even relevant since the majority of students will not gain recognition at home institution from their participation in the summer course, e.g., through the accreditation of ECTS (European Credit Transfer and Accumulation System) [14] in their original MSc or PhD studies. Students got contact with the summer school mainly through their research supervisors, that are members of the IEEE IES Technical Committee on Industrial Agents or the VDI/VDE GMA Technical Committee 5.15 on Multi-Agent Systems, but also through other researchers and the wiki cfp platform.

Fig. 4 summarizes the feedback achieved from the students regarding the satisfaction with the academic activities and the pedagogical aspects of the summer school.

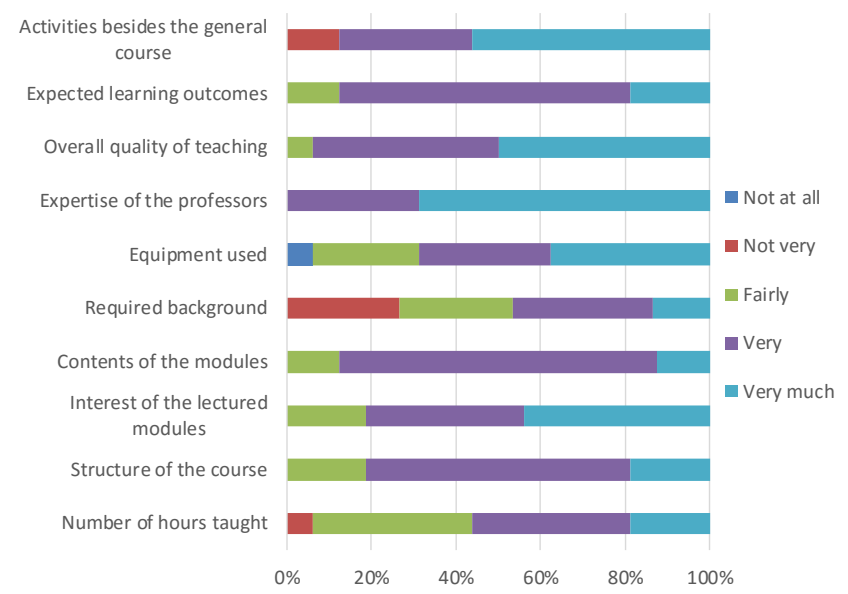

Fig. 4. Level of satisfaction with the academic and pedagogical aspects.

The analysis of the results allows to verify that all items were well scored by the students. Particularly, students recognized the overall quality of teaching ( $93,8 \%$ answered very or very much), the capabilities and expertise of the teachers $(100 \%)$, the contents of the modules $(87,5 \%)$ and the expected learning outcomes $(87,5 \%)$. Students were very or very much satisfied with the structure of the course $(81,3 \%$ ) but some (only $6,3 \%$ ) were disappointed with the number of hours taught.

The majority of students were very or very much satisfied with the equipment used $(68,8 \%$ ), but a minority (only $6,3 \%$ ) was disappointed, not with the available automation equipment but probably expecting to be provided PCs with all required software to be used during the course instead to use their own personal laptops. Some students complained with the required background for the course $(26,7 \%)$, particularly for mechanical engineers who have poor programming skills.

Students were in general very or very much satisfied with the social activities organized besides the general lecturing course $(87,5 \%)$ but some students (only 12,5\%) were expecting more.

Another important indicator to measure the success of the summer school was the very positive feedback from the participants related to the overall evaluation of the course. In fact, $25 \%$ of the students evaluated the course as excellent and $75 \%$ as good. Also important is that all attendees will 
recommend the participation of the summer school to their colleagues, and 93,8\% intend to participate in a next edition of the summer school, if organized and containing advanced topics. In this case, Fig. 5 summarizes the feedback achieved from the students regarding the topics that will be interesting to be covered by the future edition of the summer school. The most relevant topics for the students are the "Advanced JADE programming", the "Service-oriented agent based systems" and the "Agent-based modelling in Cyber-Physical Systems".

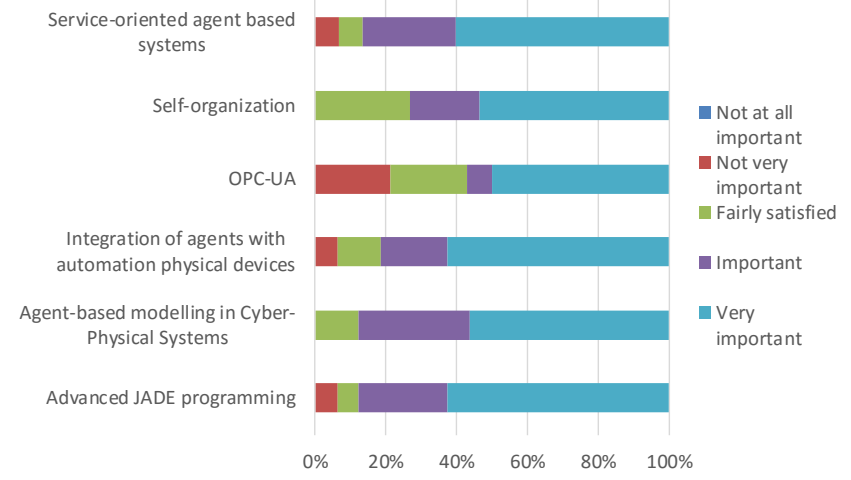

Fig. 5. Interesting topics to be covered by the future edition of the school.

An additional important conclusion is that the students recognize the importance of the participation in the learning course for their further studies / career $(80 \%)$, but only $14,3 \%$ are convinced that this will help them to find a job.

\section{B. Innovation and Impact}

The innovation of this summer school is mainly related to the hands-on practical experience on developing agent-based solutions in the context of CPS with different approaches and programming languages. The comparison of these approaches and the identification of applicability in different domains and levels of CPS systems are relevant issues.

The sharing of complementary knowledge, experiences and learning practices is another key benefit, which may be fundamental to establish and strength international academic/scientific networks aiming to develop future partnerships for project proposals submitted to funding programs, e.g., the EU Horizon 2020 program. Understanding the different learning styles facilitates the students' mobility and the introduction of new student by adapting to their traditional practices in pre-courses, discussing such specific styles to achieve awareness to both students and teachers.

Therefore, the impact for future $\mathrm{PhDs}$ as well as researchers knowing right from the beginning the agent paradigm and its different approaches, understanding the constraints and the world model behind each approach shall not be underestimated. Additionally, listening lectures of other researchers, the underlying world model and constraints are easier to be identified when compared to short talks in conferences. This is why the benefit for a researcher to attend a summer school is not limited to presenting his or her results to the community and young $\mathrm{PhDs}$ but helps to develop a deep understanding of related and sometimes even competitive approaches. Based on such deeper insights future research cooperation is facilitated.

\section{CONCLUSIONS}

This paper describes the experience gathered from the implementation of the summer school on intelligent agents in automation, technically sponsored by IEEE IES Technical Committee on Industrial Agents. The objective of the course was to provide a range of theoretical and practical knowledge in applying the agent technology in industrial automation domains, and particularly to develop complex, real industrial agent-based applications, aligned with emergent CPS context. In particular, a strong emphasis is highlighted in providing practical hands-on experience on deploying industrial agents.

The course was lectured by several experts in the industrial agent technology, which contributed to share knowledge and learning practices. The multi-disciplinary perspective allowed to have a complementary view of different technological topics. The feedback from the students showed that they were highly satisfied with the summer school, in organizational and pedagogical terms, which makes the summer school a successful experience. Students were motivated to recommended the summer school to their colleagues, but also to participate in a future edition if organized and containing advanced topics.

\section{REFERENCES}

[1] P. Leitão, S. Karnouskos, L. Ribeiro, J. Lee, T. Strasser, A. W. Colombo, "Smart agents in industrial cyber-physical systems", in Press, Proceedings of the IEEE, 2016.

[2] S. K. Khaitan, J. D. McCalley, "Design techniques and applications of cyberphysical systems: A survey", IEEE Systems Journal, vol. 9, pp. 350$365,2015$.

[3] J. Lee, B. Bagheri, H.-A. Kao, "A cyber-physical systems architecture for industry 4.0-based manufacturing systems", Manufacturing Letters, vol. 3, pp. 18-23, 2015.

[4] M. Wooldridge, N. R. Jennings, "Intelligent agents: theory and practice", The Knowledge Engineering Review, vol. 10, pp. 115-152, 1995.

[5] P. Leitão, V. Marik, P. Vrba, "Past, present, and future of industrial agent applications", IEEE Transactions on Industrial Informatics, vol. 9, pp. 2360-2372, 2013.

[6] V. Marik D. McFarlane, "Industrial adoption of agent-based technologies", IEEE Intelligent Systems, vol. 20, pp. 27-35, 2005.

[7] F. Bellifemine, G. Caire, D. Greenwood, "Developing multi-agent systems with JADE", Wiley, 2007.

[8] International Electrotechnical Commission, "IEC 61131-3: Programmable Controllers, Part 3: Programming Languages," IEC, 2003.

[9] C. Legat, D. Schütz, B. Vogel-Heuser, "Automatic generation of field control strategies for supporting (re-)engineering of manufacturing systems", Journal of Intelligent Manufacturing, vol. 25, no. 5, pp. 1101$1111,2013$.

[10] International Electrotechnical Commission, "IEC 61499: Function blocks Part 1-4", IEC, 2012.

[11] T. Strasser, M. Rooker, G. Ebenhofer, A. Zoitl, C. Sünder, A. Valentini, A. Martel, "Framework for distributed industrial automation and control (4DIAC)", Proc. 6th IEEE Intern. Conf. on Industrial Informatics (INDIN'08), pp. 283-288, 2008.

[12] L. Ribeiro, J. Barata, M. Onori, J. Hoos, "Industrial agents for the fast deployment of evolvable assembly systems", Industrial Agents: Emerging Applications of Software Agents in Industry, P. Leitão and S. Karnouskos (eds.). Elsevier, pp. 301-322, 2015.

[13] A. Rocha, G. di Orio, J. Barata, N. Antzoulatos, E. Castro, D. Scrimieri, S. Ratchev, L. Ribeiro, "An agent based framework to support plug and produce," Proc. 12 $2^{\text {th }}$ IEEE Intern. Conf. on Industrial Informatics (INDIN'14), pp. 504-510, 2014.

[14] European Commission, "European Credit Transfer and Accumulation System", available at http://ec.europa.eu/education/tools/ects en.htm (accessed on 27/02/2016). 